\title{
Publisher Correction: Protein-altering variants associated with body mass index implicate pathways that control energy intake and expenditure in obesity
}

Valérie Turcot, Yingchang Lu, Heather M. Highland, Claudia Schurmann (D), Anne E. Justice (D), Rebecca S. Fine, Jonathan P. Bradfield, Tõnu Esko, Ayush Giri D, Mariaelisa Graff, Xiuqing Guo, Audrey E. Hendricks, Tugce Karaderi, Adelheid Lempradl, Adam E. Locke (D), Anubha Mahajan, Eirini Marouli, Suthesh Sivapalaratnam, Kristin L. Young (D, Tamuno Alfred, Mary F. Feitosa, Nicholas G. D. Masca, Alisa K. Manning, Carolina Medina-Gomez (D), Poorva Mudgal, Maggie C. Y. Ng, Alex P. Reiner, Sailaja Vedantam, Sara M. Willems, Thomas W. Winkler (D), Gonçalo Abecasis, Katja K. Aben, Dewan S. Alam, Sameer E. Alharthi, Matthew Allison, Philippe Amouyel, Folkert W. Asselbergs, Paul L. Auer, Beverley Balkau, Lia E. Bang, Inês Barroso, Lisa Bastarache, Marianne Benn, Sven Bergmann, Lawrence F. Bielak, Matthias Blüher, Michael Boehnke, Heiner Boeing, Eric Boerwinkle, Carsten A. Böger, Jette Bork-Jensen, Michiel L. Bots, Erwin P. Bottinger, Donald W. Bowden, Ivan Brandslund, Gerome Breen, Murray H. Brilliant, Linda Broer, Marco Brumat, Amber A. Burt, Adam S. Butterworth, Peter T. Campbell, Stefania Cappellani, David J. Carey, Eulalia Catamo, Mark J. Caulfield, John C. Chambers, Daniel I. Chasman, Yii-Der I. Chen, Rajiv Chowdhury, Cramer Christensen, Audrey Y. Chu, Massimiliano Cocca, Francis S. Collins, James P. Cook, Janie Corley, Jordi Corominas Galbany, Amanda J. Cox, David S. Crosslin, Gabriel Cuellar-Partida, Angela D'Eustacchio, John Danesh, Gail Davies, Paul I. W. Bakker, Mark C. H. Groot, Renée Mutsert, lan J. Deary, George Dedoussis, Ellen W. Demerath, Martin Heijer, Anneke I. Hollander, Hester M. Ruijter, Joe G. Dennis, Josh C. Denny, Emanuele Di Angelantonio, Fotios Drenos, Mengmeng Du, Marie-Pierre Dubé, Alison M. Dunning, Douglas F. Easton, Todd L. Edwards, David Ellinghaus, Patrick T. Ellinor, Paul Elliott, Evangelos Evangelou, Aliki-Eleni Farmaki, I. Sadaf Farooqi, Jessica D. Faul, Sascha Fauser, Shuang Feng, Ele Ferrannini, Jean Ferrieres, Jose C. Florez, Ian Ford, Myriam Fornage, Oscar H. Franco, Andre Franke, Paul W. Franks, Nele Friedrich, Ruth Frikke-Schmidt, Tessel E. Galesloot, Wei Gan, Ilaria Gandin, Paolo Gasparini, Jane Gibson, Vilmantas Giedraitis, Anette P. Gjesing, Penny Gordon-Larsen, Mathias Gorski, Hans-Jörgen Grabe, Struan F. A. Grant, Niels Grarup, Helen L. Griffiths, Megan L. Grove, Vilmundur Gudnason, Stefan Gustafsson, Jeff Haessler, Hakon Hakonarson, Anke R. Hammerschlag, Torben Hansen, Kathleen Mullan Harris, Tamara B. Harris, Andrew T. Hattersley, Christian T. Have, Caroline Hayward, Liang He, Nancy L. Heard-Costa, Andrew C. Heath, Iris M. Heid, Øyvind Helgeland, Jussi Hernesniemi, Alex W. Hewitt, Oddgeir L. Holmen, G. Kees Hovingh, Joanna M. M. Howson, Yao Hu, Paul L. Huang, Jennifer E. Huffman, M. Arfan Ikram, Erik Ingelsson, Anne U. Jackson, Jan-Håkan Jansson, Gail P. Jarvik, Gorm B. Jensen, Yucheng Jia, Stefan Johansson, Marit E. Jørgensen, Torben Jørgensen, J. Wouter Jukema, Bratati Kahali, René S. Kahn, Mika Kähönen, Pia R. Kamstrup, Stavroula Kanoni, Jaakko Kaprio, Maria Karaleftheri, Sharon L. R. Kardia, Fredrik Karpe, Sekar Kathiresan, Frank Kee, Lambertus A. Kiemeney, Eric Kim, Hidetoshi Kitajima, Pirjo Komulainen, Jaspal S. Kooner, Charles Kooperberg, Tellervo Korhonen, Peter Kovacs, Helena Kuivaniemi, Zoltán Kutalik, Kari Kuulasmaa, Johanna Kuusisto, Markku Laakso, Timo A. Lakka, David Lamparter, Ethan M. Lange, Leslie A. Lange, Claudia Langenberg, Eric B. Larson, Nanette R. Lee, Terho Lehtimäki, Cora E. Lewis, Huaixing Li, Jin Li, Ruifang Li-Gao, Honghuang Lin, Keng-Hung Lin, Li-An Lin, Xu Lin, Lars Lind, Jaana Lindström, Allan Linneberg, Ching-Ti Liu, Dajiang J. Liu, Yongmei Liu, Ken S. Lo, Artitaya Lophatananon, Andrew J. Lotery, Anu Loukola, Jian'an Luan, Steven A. Lubitz, Leo-Pekka Lyytikäinen, Satu Männistö, Gaëlle Marenne, Angela L. Mazul, Mark I. McCarthy, Roberta McKean-Cowdin, Sarah E. Medland, Karina Meidtner, Lili Milani, Vanisha Mistry, Paul Mitchell, Karen L. Mohlke, Leena Moilanen, Marie Moitry, Grant W. Montgomery, Dennis O. Mook-Kanamori, Carmel Moore, Trevor A. Mori, Andrew D. Morris, Andrew P. Morris, Martina Müller-Nurasyid, Patricia B. Munroe, Mike A. Nalls, Narisu Narisu, Christopher P. Nelson, Matt Neville, Sune F. Nielsen, Kjell Nikus, Pål R. Njølstad, Børge G. Nordestgaard, Dale R. Nyholt, Jeffrey R. O'Connel, Michelle L. O'Donoghue, Loes M. Olde Loohuis, Roel A. Ophoff, Katharine R. Owen, Chris J. Packard, Sandosh Padmanabhan, Colin N. A. Palmer, Nicholette D. Palmer, Gerard Pasterkamp, Aniruddh P. Patel, Alison Pattie, Oluf Pedersen, Peggy L. Peissig, Gina M. Peloso, Craig E. Pennell, Markus Perola, James A. Perry, John R. B. Perry, Tune H. Pers, Thomas N. Person, Annette Peters, Eva R. B. Petersen, Patricia A. Peyser, Ailith Pirie, Ozren Polasek, Tinca J. Polderman, Hannu Puolijoki, Olli T. Raitakari, Asif Rasheed, Rainer Rauramaa, Dermot F. Reilly, Frida Renström, Myriam Rheinberger, Paul M. Ridker, John D. Rioux, Manuel A. Rivas, David J. Roberts, Neil R. Robertson,

Antonietta Robino, Olov Rolandsson, Igor Rudan, Katherine S. Ruth, Danish Saleheen, Veikko Salomaa, Nilesh J. Samani, Yadav Sapkota, Naveed Sattar, Robert E. Schoen, Pamela J. Schreiner, Matthias B. Schulze, Robert A. Scott,

Marcelo P. Segura-Lepe, Svati H. Shah, Wayne H.-H. Sheu, Xueling Sim, Andrew J. Slater, Kerrin S. Small, Albert V. Smith, Lorraine Southam, Timothy D. Spector, Elizabeth K. Speliotes, John M. Starr, Kari Stefansson, Valgerdur Steinthorsdottir, Kathleen E. Stirrups, Konstantin Strauch, Heather M. Stringham, Michael Stumvoll, Liang Sun, Praveen Surendran, Amy J. Swift, Hayato Tada, Katherine E. Tansey, Jean-Claude Tardif, Kent D. Taylor, Alexander Teumer, Deborah J. Thompson, Gudmar Thorleifsson, Unnur Thorsteinsdottir, Betina H. Thuesen, Anke Tönjes, Gerard Tromp, 
Stella Trompet, Emmanouil Tsafantakis, Jaakko Tuomilehto, Anne Tybjaerg-Hansen, Jonathan P. Tyrer, Rudolf Uher, André G. Uitterlinden, Matti Uusitupa, Sander W. Laan, Cornelia M. Duijn, Nienke Leeuwen, Jessica van Setten, Mauno Vanhala, Anette Varbo, Tibor V. Varga, Rohit Varma, Digna R. Velez Edwards, Sita H. Vermeulen, Giovanni Veronesi, Henrik Vestergaard, Veronique Vitart, Thomas F. Vogt, Uwe Völker, Dragana Vuckovic, Lynne E. Wagenknecht, Mark Walker, Lars Wallentin, Feijie Wang, Carol A. Wang, Shuai Wang, Yiqin Wang, Erin B. Ware, Nicholas J. Wareham, Helen R. Warren, Dawn M. Waterworth, Jennifer Wessel, Harvey D. White, Cristen J. Willer, James G. Wilson, Daniel R. Witte, Andrew R. Wood, Ying Wu, Hanieh Yaghootkar, Jie Yao, Pang Yao, Laura M. Yerges-Armstrong, Robin Young, Eleftheria Zeggini B, Xiaowei Zhan, Weihua Zhang, Jing Hua Zhao, Wei Zhao, Wei Zhao (1), Wei Zhou, Krina T Zondervan D, CHD Exome+ Consortium, EPIC-CVD Consortium, ExomeBP Consortium, Global Lipids Genetic Consortium, GoT2D Genes Consortium, EPIC InterAct Consortium, INTERVAL Study, ReproGen Consortium, T2D-Genes Consortium, The MAGIC Investigators, Understanding Society Scientific Group, Jerome I. Rotter, John A. Pospisilik (D), Fernando Rivadeneira (D), Ingrid B. Borecki, Panos Deloukas (D), Timothy M. Frayling, Guillaume Lettre, Kari E. North, Cecilia M. Lindgren, Joel N. Hirschhorn and Ruth J. F. Loos (i)

Correction to: Nature Genetics https://doi.org/10.1038/s41588-017-0011-x, published online 22 December 2017.

In the HTML version of this article initially published, the author groups 'CHD Exome+ Consortium', 'EPIC-CVD Consortium', 'ExomeBP Consortium, 'Global Lipids Genetic Consortium,' 'GoT2D Genes Consortium, 'EPIC InterAct Consortium', 'INTERVAL Study', 'ReproGen Consortium, 'T2D-Genes Consortium,' 'The MAGIC Investigators' and 'Understanding Society Scientific Group' appeared at the end of the author list but should have appeared earlier in the list, after author Krina T. Zondervan. The errors have been corrected in the HTML version of the article.

\section{Publisher Correction: BRD4 interacts with NIPBL and BRD4 is mutated in a Cornelia de Lange-like syndrome}

Gabrielle Olley, Morad Ansari, Hemant Bengani, Graeme R. Grimes, James Rhodes, Alex von Kriegsheim, Ana Blatnik, Fiona J. Stewart, Emma Wakeling, Nicola Carroll, Alison Ross, Soo-Mi Park, Deciphering Developmental Disorders Study, Wendy A. Bickmore $\mathbb{B}$, Madapura M. Pradeepa (1) and David R. FitzPatrick (D)

Correction to: Nature Genetics https://doi.org/10.1038/s41588-018-0042-y, published online 29 January 2018.

In the HTML version of this article initially published, the author group 'Deciphering Developmental Disorders Study' appeared at the end of the author list but should have appeared in the fourth-to-last position. The error has been corrected in the HTML version of the article.

Published online: 3 June 2019

https://doi.org/10.1038/s41588-019-0448-1

\section{Publisher Correction: Multiancestry genome-wide association study of 520,000 subjects identifies 32 loci associated with stroke and stroke subtypes}
Rainer Malik, Ganesh Chauhan, Matthew Traylor (D), Muralidharan Sargurupremraj, Yukinori Okada (D), Aniket Mishra, Loes Rutten-Jacobs D, Anne-Katrin Giese, Sander W. van der Laan D, Solveig Gretarsdottir, Christopher D. Anderson, Michael Chong, Hieab H. H. Adams (D), Tetsuro Ago, Peter Almgren (D), Philippe Amouyel (D), Hakan Ay, Traci M. Bartz, Oscar R. Benavente, Steve Bevan, Giorgio B. Boncoraglio, Robert D. Brown Jr, Adam S. Butterworth, Caty Carrera, Cara L. Carty, Daniel I. Chasman, Wei-Min Chen, John W. Cole, Adolfo Correa D, Ioana Cotlarciuc, Carlos Cruchaga (D), John Danesh, Paul I. W. de Bakker, Anita L. DeStefano, Marcel den Hoed (D), Qing Duan, Stefan T. Engelter, Guido J. Falcone, Rebecca F. Gottesman, Raji P. Grewal, Vilmundur Gudnason, Stefan Gustafsson (D, Jeffrey Haessler, Tamara B. Harris, Ahamad Hassan, Aki S. Havulinna D, Susan R. Heckbert, Elizabeth G. Holliday, George Howard, Fang-Chi Hsu, Hyacinth I. Hyacinth, M. Arfan Ikram (D), Erik Ingelsson, Marguerite R. Irvin, Xueqiu Jian, Jordi Jiménez-Conde, Julie A. Johnson, J. Wouter Jukema D, Masahiro KanaiD, Keith L. Keene, Brett M. Kissela, Dawn O. Kleindorfer, Charles Kooperberg, Michiaki Kubo, Leslie A. Lange, Carl D. Langefeld, Claudia Langenberg, Lenore J. Launer, Jin-Moo Lee, Robin Lemmens, Didier Leys, Cathryn M. Lewis (D), Wei-Yu Lin D, 